Supplement of Geosci. Model Dev., 12, 3419-3438, 2019

https://doi.org/10.5194/gmd-12-3419-2019-supplement

(C) Author(s) 2019. This work is distributed under

the Creative Commons Attribution 4.0 License.

(c) (1)

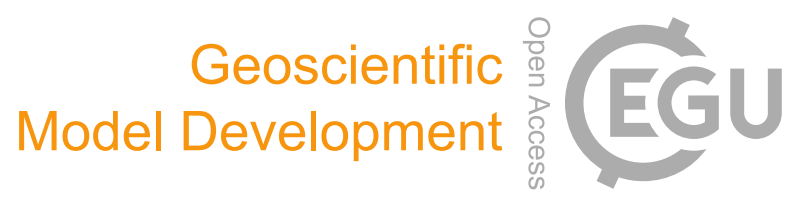

Supplement of

\title{
Climate projections of a multivariate heat stress index: the role of downscaling and bias correction
}

\author{
Ana Casanueva et al. \\ Correspondence to: Ana Casanueva (ana.casanueva@unican.es)
}

The copyright of individual parts of the supplement might differ from the CC BY 4.0 License. 


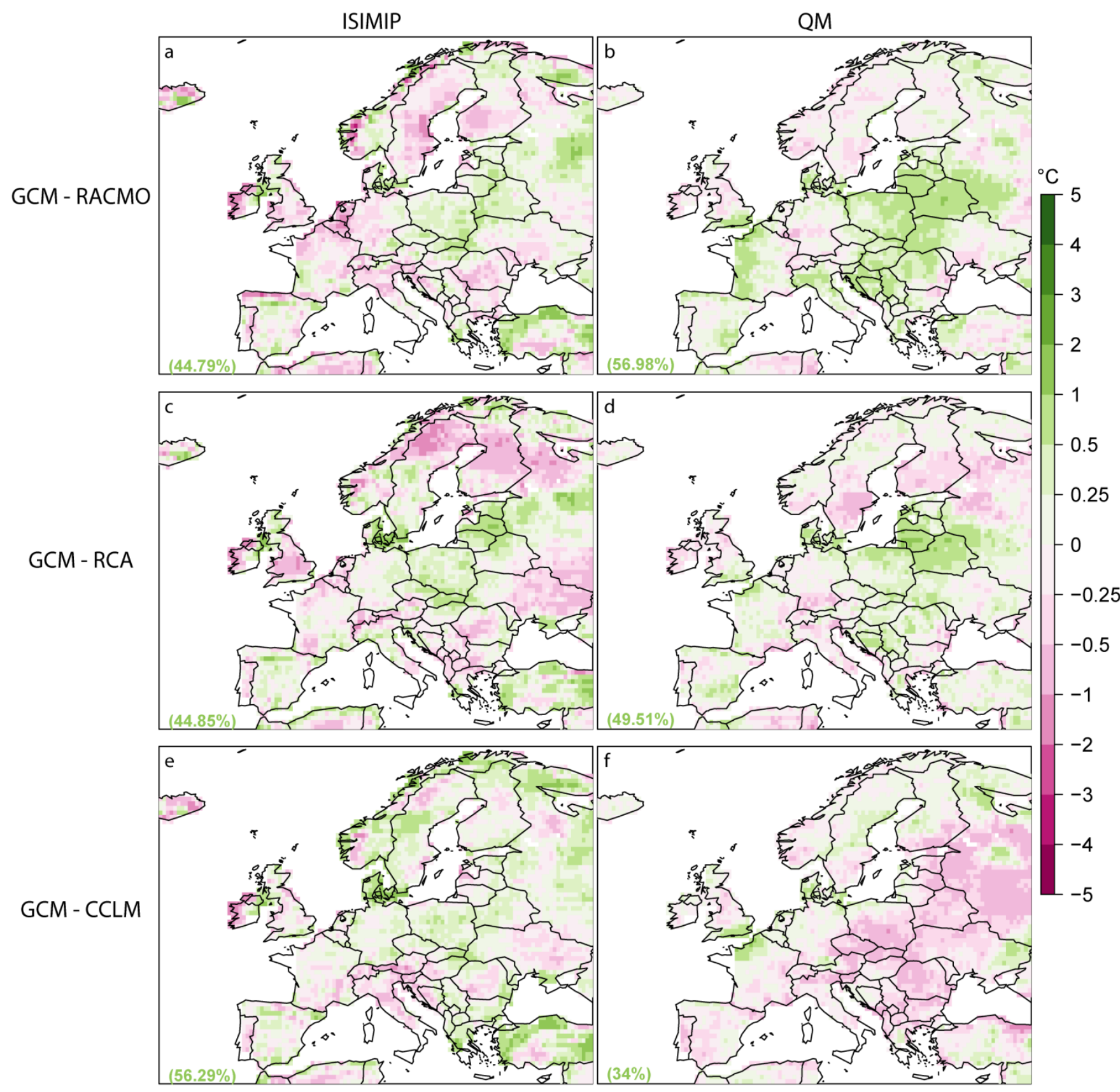

Figure S 1: Added value of high resolution after bias correction for WBGTp99. The panels show the difference between the biases (in absolute value) of the GCM minus the RCMs (EUR-011) after the two BC methods (ISIMIP -left panel- and QM -right panel). Thus, greenish colours mean added value of the RCM after BC with respect to the bias-corrected GCM. The numbers in brackets depict the percentage of grid boxes where the bias-corrected RCM improves on the bias-corrected GCM. 


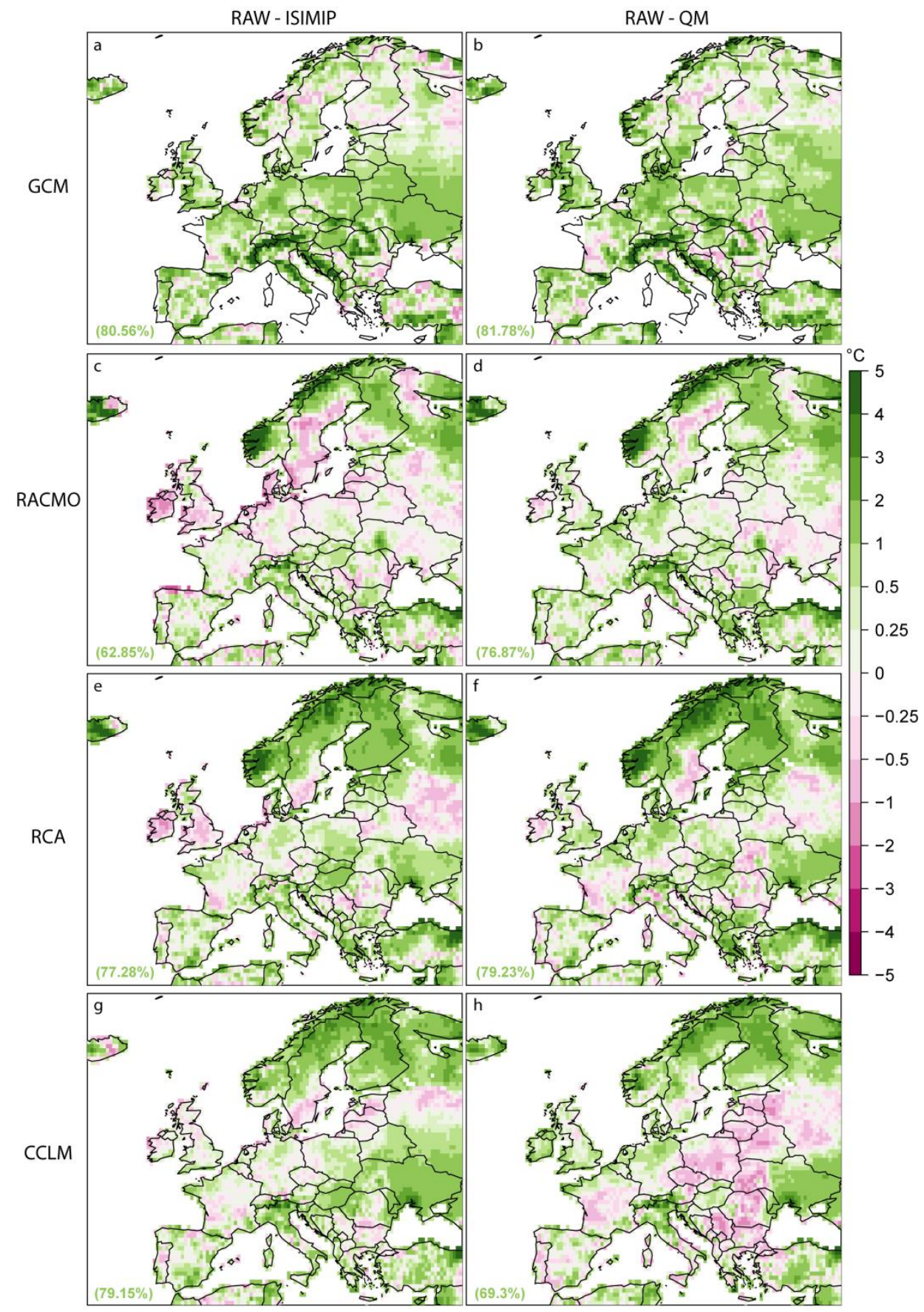

Figure S 2: Added value of bias correction for WBGTp99. The panels show the difference between the biases (in absolute value) of the raw minus the bias-corrected data (for the GCM and the RCMs-EUR-011 in rows) for the two BC methods (ISIMIP -left panel- and QM -right panel). Thus, greenish colours mean added value of the bias-corrected data with respect to the raw counterpart. The numbers in brackets depict the percentage of grid boxes where the bias-corrected data improves on the raw. 

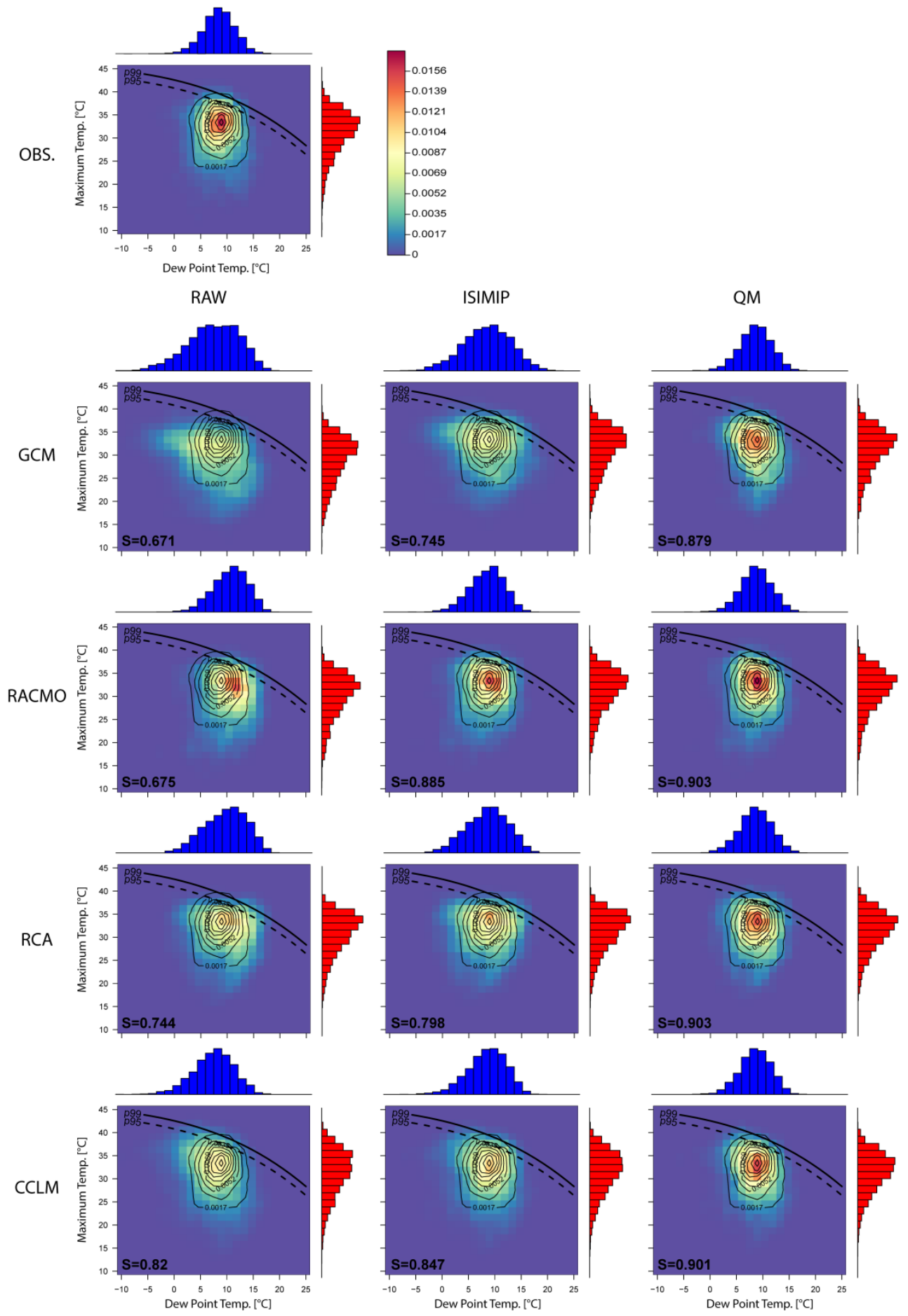

Figure S 3: As Fig.5 but for the grid box over Madrid. 

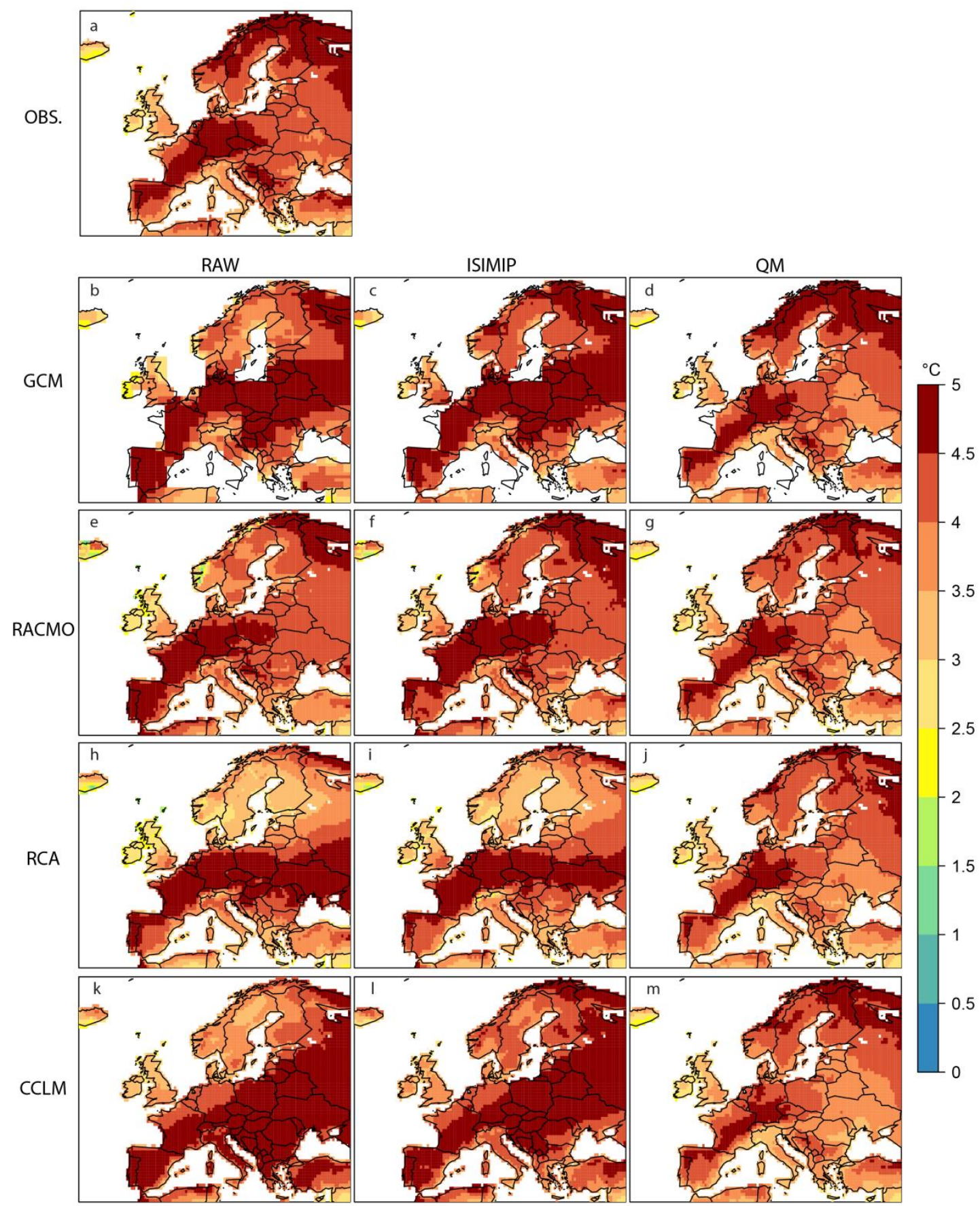

Figure S 4 Standard deviation of the distribution of daily maximum temperatures (1981-2010, JJA). Results for the observations (a), GCM (b-d) and three RCMs-EUR11 (e-m). Raw and bias-corrected data are depicted in columns. 

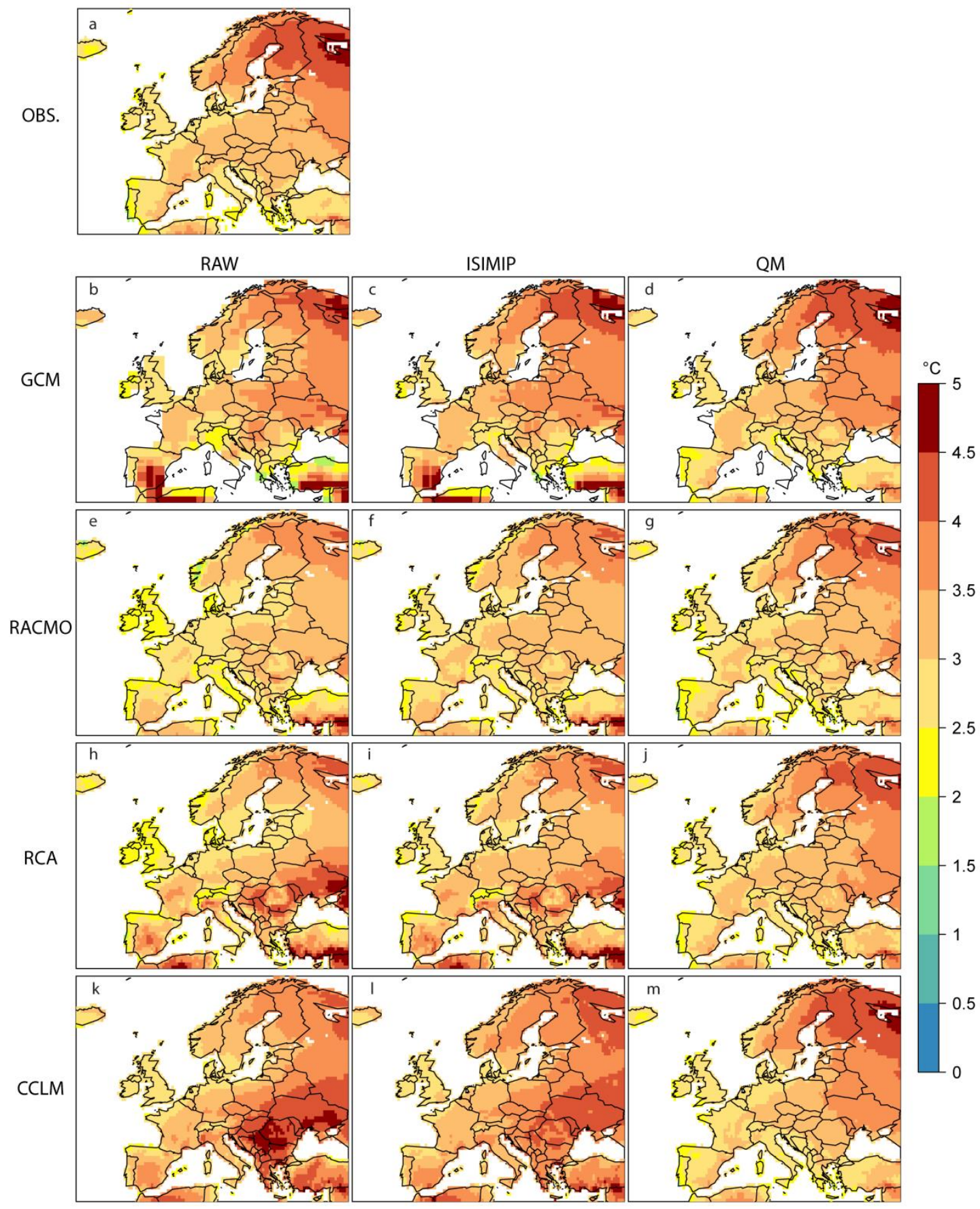

Figure S 5 As Fig.S4, but for daily mean dew point temperature. 


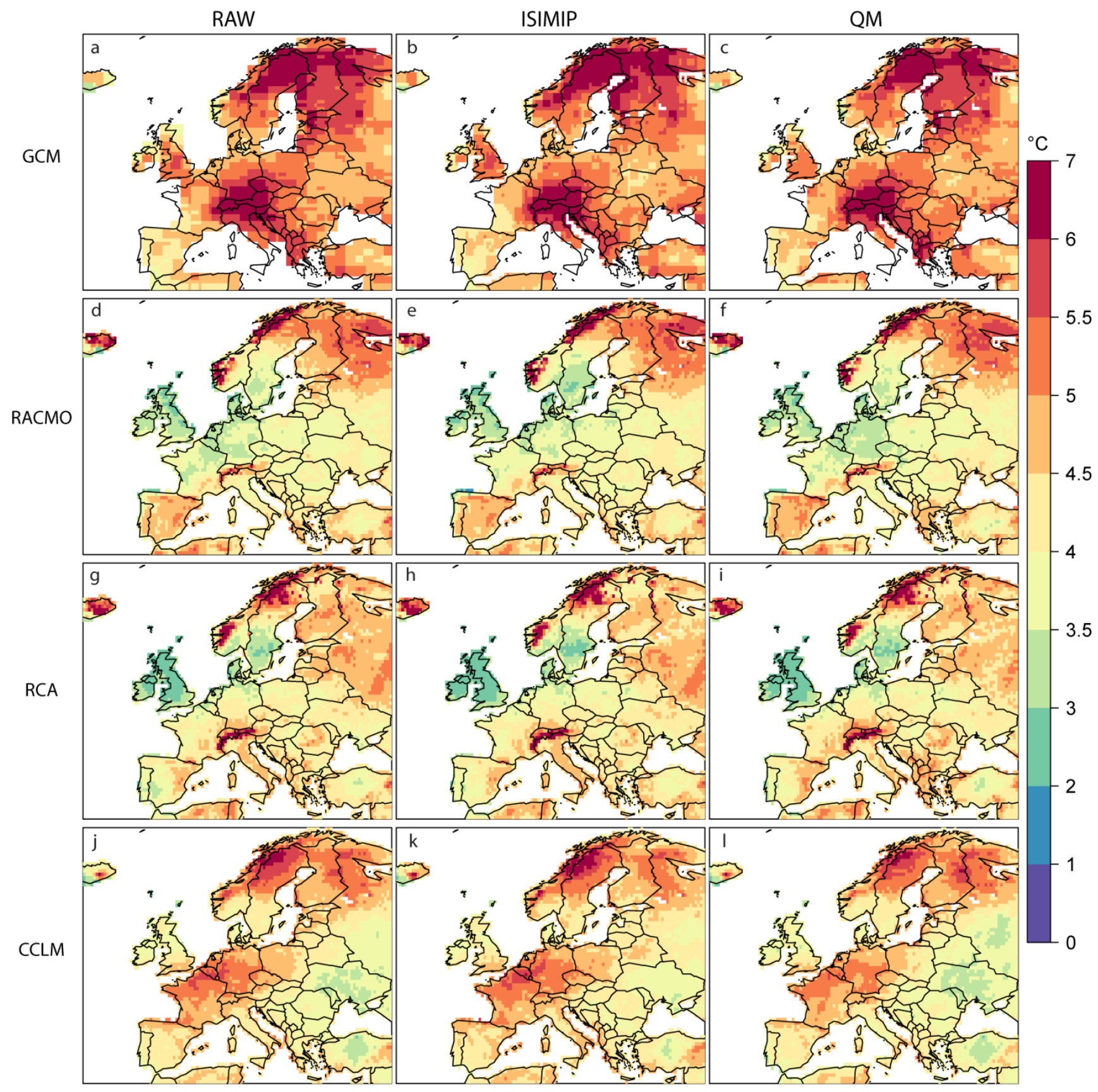

Figure S 6: As Fig.7 but for the climate change signal of the WBGTp99. 